\title{
New Understandings of Biocorrosion Mechanisms and their Classifications
}

\section{Tingyue $\mathbf{G u}^{*}$}

Department of Chemical and Biomolecular Engineering, Institute for Corrosion and Multiphase Technology, Ohio University, Athens, OH, USA

Microbes were first associated with corrosion as early as 1910 [1]. However, much confusion remains in biocorrosion mechanisms because their investigation involves multidisciplinary knowledge in several diverse research areas such as bio-electrochemistry, microbiology, corrosion engineering and chemical engineering. Billions of dollars are lost to biocorrosion each year in the US alone [2]. The Alaska pipeline leak that caused a major spike in the world regarding oil prices in the spring of 2006, heightened attentions on biocorrosion. As a consequence of depleting oil reserves and high oil prices, water flooding is more frequently used to increase reservoir pressure. The water used in the operation often introduces nutrients and microbes into the reservoirs. Microbial activities are now major concerns in oil and gas production and transportation. As infrastructures are aging, biocorrosion is becoming more often a risk factor in many industrial operations such as oil and gas transportation, water utilities, and power plant cooling systems. An additional risk factor for oil transportation lines is that pipe wall water-wetting, instead of oil-wetting, is becoming a more common flow condition and this promotes the diversity and growth of microbes, leading to faster pipe failures.

Many different terms have been used to describe corrosion caused or induced by microbes, including biocorrosion, microbial corrosion, and microbiologically influenced/induced corrosion (MIC). They have different connotations. Biocorrosion and microbial corrosion tend to hint that the microbes are the main cause of the corrosion, while MIC suggests an involvement of microbes that may or may not be direct. The words "influenced" and "induced" are inherently ambiguous. Some people tend to blame corrosion problems for which they do not have clear answers (even before conventional corrosion mechanisms are thoroughly investigated) to MIC merely because microbes are found at corrosion sites. Field systems are not sterile; the presence of microbes does not necessarily prove that microbes play any significant roles in the corrosion process. It is unfortunate that MIC forensics does not yet have the same rigor as that in the hunting of an unknown pathogen by medical researchers who rely on the Koch's postulates. This means that many reported "MIC cases" are rather speculative because no efforts have been made to reproduce the corrosion. Most laboratory investigations, especially mechanistic investigations, clearly focus on microbes as the primary cause of corrosion, rather than $\mathrm{CO}_{2}$ or other corrosion factors. To avoid ambiguity, the term 'microbiologically inflicted corrosion" may be used for such a situation to preserve the popular acronym MIC [3].

In corrosion research, one must identify the terminal electron acceptor. In many corrosion systems, such as oil pipelines, oxygen is removed by an oxygen scavenger or flash rust, or even a top-layer aerobic biofilm. However, anaerobic corrosion remains a threat. Anaerobic iron corrosion is the result of the loss of electrons by elemental iron $\left(\mathrm{Fe}^{0}\right)$ that releases soluble ferrous ion $\left(\mathrm{Fe}^{2+}\right)$ as shown below. The standard potentials at $\mathrm{pH} 7\left(\mathrm{E}^{\circ}\right)$ in Reaction (1) and elsewhere in this work were taken from Thuar et al. [4].

$$
\mathrm{Fe} \rightarrow \mathrm{Fe}^{2+}+2 \mathrm{e}^{-} \text {(Iron oxidation) } \quad \mathrm{E}^{\mathrm{o}^{\prime}}=+447 \mathrm{mV}
$$

In the absence of oxygen, the electrons must be accepted by a nonoxygen oxidant. Sulfate Reducing Bacteria (SRB) are most frequently found to the cause of biocorrosion. SRB respiration typically utilizes sulfate as the electron acceptor. Some SRB strains can also utilize sulfur, thiosulfate, or even $\mathrm{CO} 2$ as oxidants [4]. The following sulfate reduction reaction is a simplified presentation of dissimilatory sulfate reduction,

$$
\mathrm{SO}_{4}^{2-}+9 \mathrm{H}^{+}+8 \mathrm{e}^{-} \rightarrow \mathrm{HS}^{-}+4 \mathrm{H}_{2} \mathrm{O} \quad \mathrm{E}^{\mathrm{o}^{\prime}}=-217 \mathrm{mV}
$$

The actual sulfate reduction reactions involve many steps. For example, in Desulfovibrio vulgaris (a common SRB), the adenosine phosphosulfate (APS) pathway is used [4]. Although Reaction (2) shows consumption of proton, the actual dissimilatory sulfate reduction for $D$. vulgaris does not cause a net change in scalar or vectorial protons [5]. The redox reaction coupling Reactions (1) and (2) are thermodynamically favorable, because the combined redox reaction has a positive potential of $230 \mathrm{mV}$, which corresponds to a negative Gibbs free energy based on the following equation,

$$
\Delta \mathrm{G}^{\circ}=-\mathrm{nF} \Delta \mathrm{E}^{\prime}
$$

$\mathrm{Fe}^{0}$ as a fuel (or electron donor) in Reaction (1) is as energetic as lactate that is a favor organic carbon for many SRB. This is because $\mathrm{CO}_{2}$ plus acetate/lactate has a standard reduction potential of $-430 \mathrm{mV}$, close to that of $-447 \mathrm{mV}$ for $\mathrm{Fe}^{2+} / \mathrm{Fe}^{0}$. The thermodynamically favorable redox reaction combining Reactions (1) and (2) moves forward only with biocatalysis because it is a kinetically retarded reaction. Like other microbes, SRB rely on biocatalyzed redox reactions such as lactate oxidation coupled with sulfate reduction to provide energy in their metabolism. If there is local shortage of organic carbon, which can happen at the bottom of a biofilm due to mass transfer resistance, SRB can switch to $\mathrm{Fe}^{0}$ as electron donor for its respiration of sulfate [6].

In addition to SRB, other microbes such as acid producing bacteria (APB) and methanogens are also known to be corrosive. There are at least two distinct types of anaerobic biocorrosion based on anaerobic metabolism [3]. Type I is caused by electrogenic microbes (and microbes that use $\mathrm{H}_{2}$ as an electron carrier) that perform respiration metabolism, such as SRB. They attack carbon steel, stainless steel and some other non-noble metals that have sufficiently negative reduction potentials on purpose using enzyme catalysis in the cytoplasm for

*Corresponding author: Tingyue Gu, Professor, Department of Chemical and Biomolecular Engineering, Ohio University, Athens, OH 45701-2979, USA, E-mail: gu@ohio.edu

Received August 28, 2012; Accepted August 30, 2012; Published August 31, 2012

Citation: Gu T (2012) New Understandings of Biocorrosion Mechanisms and their Classifications. J Microbial Biochem Technol 4: iii-vi. doi:10.4172/19485948.1000e107

Copyright: (c) $2012 \mathrm{Gu}$ T. This is an open-access article distributed under the terms of the Creative Commons Attribution License, which permits unrestricted use, distribution, and reproduction in any medium, provided the original author and source are credited 
reduction of an oxidant (such as sulfate), because these metals are electron donors. Type II is caused by fermentative microbes, such as APB that secrete corrosive metabolites. These secreted oxidants are oxidized on the metal surface extracellularly. Fermentative metabolism does not require external electron acceptors and it produces metabolites that usually include relatively large amounts of organic acids. Proton reduction at a sufficiently acidic $\mathrm{pH}$ can be coupled with iron oxidation to form a thermodynamically favorable redox reaction that is kinetically not retarded. This is not different from organic acid attack in conventional chemical corrosion such as acetic acid (HAc) attack in oil pipelines [7]. Undissociated organic acids such as free HAc may also be reduced directly in addition to serving as a proton reservoir, although this mechanism is now disputed. Both Types I and II biocorrosion mechanisms are electrochemical. An additional Type III biocorrosion may be defined for microbial attack of an extracellular organic substance such as polyurethane and its organic plasticizer for the purpose of harvesting organic carbon and energy by the microbes [8]. This type of non-electrochemical corrosion is better known as biodegradation. The microbes involved can be aerobic or anaerobic. There may be additional types of biocorrosion, especially in biofilm attacks in an open body of water with dissolved oxygen as the possible terminal electron acceptor.

Sessile cells in biofilms rather than planktonic cells are directly responsible for biocorrosion. In Types II and III biocorrosion, a biofilm provides a local environment with corrosive chemicals that are much more concentrated because of higher local cell concentrations. For Type I biocorrosion, extracellular electrons released by iron oxidation must be transported to the cytoplasm for oxidant reduction. Unlike ions, electrons cannot "swim" in water. This means planktonic cells cannot contribute directly to Type I biocorrosion, because they cannot take electrons from a metal surface through an aqueous liquid medium. This is why sessile cells in a biofilm rather than planktonic cells directly cause Type I biocorrosion. Several electron transport mechanisms have been illustrated by microbial fuel cell (MFC) researchers who actively hunt for electrogenic biofilms to maximize electricity output [9]. They fall into two major categories: Direct electron transport (DET) and Mediated electron transport (MET). Direct electron transport relies on membrane bound electron transport proteins such as c-type cytochromes and heme proteins and pili (also known as nanowires) to pass electrons from outside the cells to the cytoplasm (as in biocorrosion or in MFCs with biocathodes) or vice versa (as in MFCs with bioanodes). In the case of electron transport proteins, the cell walls must be in direct contact with a conductive surface such as an iron surface. This means that only a monolayer of cells are directly corrosive in such as situation. When pili are used for electron transport, More than one layer of cells may be involved and directly cause corrosion. Sherar et al. [10] noticed that when their SRB-dominated field biofilm consortium was cultured in the absence of organic carbon, SRB cells formed multiple pili linking the cell wall and an iron surface. This suggests that the cells actively created the pili in order to transport the electrons from $\mathrm{Fe}^{0}$ oxidation during carbon starvation [6]. MET relies on redox active electron mediators as electron shuttles. Many mediators have been discovered in MFC research [9]. It has been discovered that an electron mediator could be used to "promote" biocorrosion in laboratory investigations [11]. In Type I biocorrosion involving methanogens and $\mathrm{H}_{2}$-consuming $\mathrm{SRB}$, molecular hydrogen can be used as an electron carrier. Methanogens and hydrogenasepositive $\mathrm{SRB}$ are capable of utilizing $\mathrm{H}_{2}$ as an energy source. $\mathrm{H}_{2}$ happens to be a common corrosion product in conventional chemical corrosion such as $\mathrm{CO}_{2}, \mathrm{H}_{2} \mathrm{~S}$, proton and organic acid attacks. It acts as an electron carrier in these microbes. The electrons released by iron oxidation are absorbed by protons to form $\mathrm{H}_{2}$. $\mathrm{H}_{2}$ oxidation releases electrons are utilized for the reduction reaction while protons are recycled.

Gu et al. [12] proposed a mechanistic biocorrosion model based on a theory known as Biocatalytic Cathodic Sulfate Reduction (BCSR). It skips the detailed species-dependent electron transport events linking Reactions (1) and (2) by introducing a calibrated electrochemical parameter termed "biofilm aggressiveness." It is actually the exchange current density for sulfate reduction in the Butler Volmer equation for charge transfer. Thus, this model can be used for all forms of electron transport mechanisms in Type I biocorrosion by SRB unlike the classical cathodic depolarization theory [13] that can only be used for hydrogenase-positive SRB. Hydrogenase enzymes in SRB are proteins that help transport extracellular electrons to the SRB cytoplasm via hydrogen oxidation and proton reduction. $\mathrm{H}_{2}$ acts like an electron shuttle. In the BCSR theory, there is no true physical cathode implied. The cathodic reaction merely refers to the sulfate reduction reaction that happens in the SRB cytoplasm under biocatalysis. This theory is readily expanded analogously to the Biocatalytic Cathodic Nitrate Reduction (BCNR) theory for Type I biocorrosion caused by electrogenic nitrate/ nitrite reducing bacteria (NRB) [3].

All the anaerobic respiring microbes involved in Type I biocorrosion may be collectively called "XRB," in which X represents an oxidant such as sulfate, nitrate/nitrite and $\mathrm{CO}_{2}$, while $\mathrm{B}$ means bugs (microbes) including bacteria, archaea and eucaryotes [3]. XRB corrosion is motivated by XRB's utilization of $\mathrm{Fe}^{0}$ as an energy source. Experiments already proved that $\mathrm{Fe}^{0}$ can serve as the sole energy source for some SRB, NRB and methanogens $[14,15]$. XRB do not "eat" metals. They just use them as electron donors. Xu and Gu [6] demonstrated that starting from mature $D$. vulgaris biofilms grown under the same conditions, the biofilms that were subsequently starved of organic carbon were more aggressive against carbon steel despite the fact that starvation could weaken the biofilms. $\mathrm{Fe}^{0}$ is treated by XRB as a fuel molecule much like an organic carbon such as lactate or $\mathrm{H}_{2}$ because $\mathrm{Fe}^{0}$ is an electron donor, and the standard reduction potential of $\mathrm{Fe}^{2+} / \mathrm{Fe}^{0}(-447 \mathrm{mV})$ is more negative than those for sulfate reduction, nitrate/nitrate reduction and $\mathrm{CO}_{2}$ reduction. XRB share a common feature that is their lifestyle of anaerobic respiration, i.e., they utilize an external electron acceptor. The reduction of the oxidant in the cytoplasm requires enzyme catalysis. This means Type I biocorrosion is intentional because it involves active participation of the microbes through biocatalysis. An electrogenic biofilm (or a biofilm capable of using $\mathrm{H}_{2}$ as an electron carrier) is required because $\mathrm{Fe}^{0}$ is insoluble and the electrons from extracellular $\mathrm{Fe}^{0}$ oxidation must rely on an elaborate electron transport chain to enter the cytoplasm where they are consumed for a reduction reaction such as sulfate, nitrate/nitrite or $\mathrm{CO}_{2}$ reduction.

It should be pointed out that unlike NRB and methanogen corrosion, SRB corrosion has complications from the release of $\mathrm{H}_{2} \mathrm{~S}$ and the formation of iron sulfide films. There are several different forms of iron sulfide films. Among them, Mackinawite is quite protective. It is the reason why a high $\mathrm{H}_{2} \mathrm{~S}$ concentration actually passivates an iron surface against further corrosioin. Some researchers [16] suggested that FeS film is the cause of SRB corrosion. This is controversial. First of all, $\mathrm{S}^{2-}$ is the end of reduction state for the sulfur element. It is not an electron acceptor. This means the FeS film at most serves a conductive mineral film between the iron and biofilm (rather than an 
electron acceptor), which may impact biofilm adherence to the iron surface. Sulfate is nonetheless the terminal electron acceptor. An iron sulfide film could be the product of SRB corrosion rather than the cause. Accepting FeS film as the cause of SRB corrosion could not provide any hint on why NRB are corrosive because the latter does not involve sulfide at all. In SRB experiments designed for biocorrosion mechanism investigations, one must be extremely carefully that no oxidant is introduced into the system inadvertently. $\mathrm{NaS}$ is a strong oxygen scavenger. One must not use this hygroscopic chemical that absorbs moisture and reacts readily with room air to adjust sulfide and bisulfide concentrations in the experiments. This is because oxygen would quickly react with $S^{2-}$ to produce various sulfur containing oxidants that will act as electron acceptors. Another caution in SRB experiments is that, sulfide readily poisons common $\mathrm{pH}$ and dissolved oxygen electrodes, resulting in erroneous readings. This means that an abnormally low $\mathrm{pH}$ underneath an SRB biofilm must be substantiated using sulfide-proof $\mathrm{pH}$ measurements. If there is an abnormal acidic $\mathrm{pH}$ reading, other mechanisms such as Type II biocorrosion due to fermentative metabolism may be involved.

Some researchers found that exopolymers in several biofilms are corrosive. This is not a surprise at all, because these exopolymers contain uronic acids that are electron acceptors [3]. This falls into Type II biocorrosion. In Type II biocorrosion, a "well-fed" biofilm produces more corrosive metabolites, and thus more corrosive. All the APB cells in a biofilm will contribute to a locally more acidic environment underneath the biofilm. Planktonic cells may indirectly contribute by lowering the bulk-fluid $\mathrm{pH}$ and thus allowing the biofilm to retain local acidity better.

The majority of microbes are incapable of transporting extracellular electrons. However, non-electrogenic microbes (or microbes that do not use $\mathrm{H}_{2}$ as an electron carrier) may live in a synergistic biofilm consortium and contribute to Type I biocorrosion indirectly by doing things such as secreting volatile fatty acids (VFAs) that are favored organic nutrients for SRB. In field analysis of biofilm samples, these "non-corrosive" microbes usually occupy the bulk of the biofilm, while the corrosive microbes may occupy only the bottom of biofilm on the metal surface (i.e., their "job site"). Thus, sampling and imaging of the biofilm should pay special attention to these electrogenic cells at the very bottom. This means that the current practice of blaming the dominant microbes in a biofilm for biocorrosion may miss the true "villain" when Type I biocorrosion is the case, because the direct culprits may be only a tiny fraction of the biofilm while the dominant microbes may at most be the "accomplice" in a synergistic biofilm community.

One also must be aware that Type I biocorrosion involves active electron transport for energy production. Using an active electrochemical method such as Electrical Resistance (ER) and Linear Polarization Resistance (LPR) for biocorrosion measurement or biofilm detection is questionable because such methods may interfere with the native electron transport/corrosion process [17]. In fact, the external electric voltage applied in impressed current cathodic protection sometimes promotes SRB growth inadvertently [18]. For Type II corrosion, ER and LPR may interfere with biofilm metabolism, but the corrosion event (e.g., proton reduction) happens extracellularly just like in conventional chemical corrosion. As long as the electrochemical method used does not change the secretion rate of corrosive metabolites, the remaining concern is merely whether the method could cope with a biofilm that is not a resistive mineral film.

Although, hundreds of corrosion related microbes have been reported in the literature, rigorous systematic investigations of biocorrosion mechanisms are still lacking. This hampers MIC forensics and the decision-making of when costly pigging and biocide treatments should be done. Instead of hunting for additional corrosive microbes, it is more urgent to investigate the biocorrosion mechanisms of known microbes. The effect of biofilm synergy on biocorrosion has not been seriously investigated at all because researchers haven't even thoroughly understood pure-strain systems that are relatively much less complicated. In a truly syntrophic biofilm community, in addition to nutrient sharing, energy sharing in a biofilm consortium is particularly important. This is true especially for Type I biocorrosion because its sole purpose is energy harvest. One should also pay attention to the secretion of electron mediators by non-corrosive microbes in the promotion of Type I biocorrosion caused by corrosive microbes. Chemical and electrochemical assays can be developed to detect these mediators. Understanding electron transfer in biofilms is critical. Fortunately, the recently intensified MFC research has accumulated a wealth of such knowledge that can be adopted after modifications. The frequent appearance of methanogens in oil and gas industry biofilm consortia should not be a surprise because $\mathrm{H}_{2}$ released by iron corrosion is an energy molecule for them. As an electron carrier, $\mathrm{H}_{2}$ is an interspecies energy transfer molecule for biofilm communities [19]. In addition to $\mathrm{H}_{2}$, formate may also serve as an electron shuttle for biofilm communities involving organic carbon degrading microbes and methanogens [20] or $\mathrm{H}_{2}$ utilizing SRB. In MFC research, it is often observed that a biofilm consortium on a bioanode usually performs much better at donating electrons (released from organic carbon oxidation) to the anode than a pure-stain biofilm. In biocorrosion, more severe attacks may come from a synergistic biofilm consortium capable of removing electrons from a metal surface more efficiently.

\section{References}

1. Gaines HA (1910) Bacterial activity as a corrosion induced in the soil. $J$ Engineer Ind Chem 2: 128-130

2. Walsh D, Pope D, Danford M, Huff T (1993) The effect of microstructure on microbiologically influenced corrosion. JOM-J Min Met Mat S 45: 22-30.

3. Gu T (2012) Can acid producing bacteria be responsible for very fast MIC pitting? Corrosion 2012, Paper No. C2012-0001214, NACE International Houston, TX.

4. Thauer RK, Stackebrandt E, Hamilton WA (2007) Energy metabolism phylogenetic diversity of sulphate-reducing bacteria. In LL Barton, WA Hamilton, (edn), Sulphate-Reducing Bacteria: Environmental and Engineered Systems. Cambridge University Press, UK, 1-37.

5. Peck HD (1993) Bioenergetic strategies of the sulfate-reducing bacteria. In JM Odom, R Singleton, Jr (Eds.), The Sulfate- Reducing Bacteria: Contemporary Perspectives. Springer-Verlag, Berlin-New York, 41-76.

6. Xu D, Gu T (2011) Bioenergetics explains when and why more severe MIC pitting by SRB can occur. Corrosion 2011 Corrosion 2011, Paper No. 11426 , NACE International, Houston, TX.

7. Joosten MW, Kolts J, Hembree JW (2002) Organic acid corrosion in oil and gas production. Corrosion 2002, Paper No. 02294, NACE International, Houston, TX.

8. Gu JD (2003) Microbiological deterioration and degradation of synthetic polymeric materials: recent research advances. Int Biodeter Biodegr 52: 69-91.

9. Du Z, Li H, Gu T (2007) A state of the art review on microbial fuel cells: A promising technology for wastewater treatment and bioenergy. Biotechnol Adv 25: 464-482.

10. Sherar BWA, Power IM, Keech PG, Mitlin S, Southam G, et al. (2011) Characterizing the effect of carbon steel exposure in sulfide containing solutions to microbially induced corrosion. Corrosion Science 53: 955-960. 
Citation: Gu T (2012) New Understandings of Biocorrosion Mechanisms and their Classifications. J Microbial Biochem Technol 4: iii-vi. doi:10.4172/1948-5948.1000e107

11. Gu T, Xu D (2010) Demystifying MIC Mechanisms. Corrosion 2010, Paper No. 10213, NACE International, Houston, TX.

12. Gu T, Zhao K, Nesic S (2009) A new mechanistic model for MIC based on a Biocatalytic Cathodic Sulfate Reduction Theory. Corrosion 2009, Paper No. 09390, NACE International, Houston, TX.

13. Von Wolzogen KCAH, Van der VLS (1934) The graphitization, of cast iron as an electrochemical process in anaerobic soils. Water 18: 147-165.

14. Dinh HT, Kuever J, Mussmann M, Hassel AW, Stratmann M, et al. (2004) Iron corrosion by novel anaerobic microorganisms. Nature 427: 829-832.

15. Till BA, Weathers LJ, Alvarez PJJ (1998) Fe(0)-supported autotrophic denitrification. Environmental Science \& Technology 32: 634-639.
16. King RA, Miller JDA (1971) Corrosion by the Sulphate-reducing Bacteria Nature 233: 491-492.

17. Janknecht P, Melo LF (2003) Online biofilm monitoring. Rev Environ Sci Biotechnol 2: 269-283

18. de Romero M, de Rincon O, Ocando L (2009) Cathodic protection efficiency in the presence of SRB: State of the art. Corrosion 2009, Paper No. 09407, NACE International, Houston, TX

19. James GA, Beaudette L, Costerton JW (1995) Interspecies bacterial interactions in biofilms. J Ind Microbiol Biot 15: 257-262.

20. Morita M, Malvankar NS, Franks AE, Summers ZM, Giloteaux L, et al. (2011) Potential for direct interspecies electron transfer in methanogenic wastewater digester aggregates. mBio 2: e00159-e00211. 